\title{
Enhanced Intestinal Permeability of Silymarin by Natural Products as Bioenhancers - Assessment by Ex-Vivo Non- Everted Rat Gut Sac Study
}

\section{Javed S ${ }^{1}$, Kohli $\mathrm{K}^{1 *}$ and Ahsan W2}

1Department of Pharmaceutics, Jamia Hamdard, India

2Department of Pharmaceutical Chemistry, College of Pharmacy, Jazan University, Saudi Arabia

*Corresponding Author: Kanchan Kohli, Department of Pharmaceutics, Faculty of Pharmacy, Jamia Hamdard, New Delhi 110062, India, Email: kanchankohli2010@gmail.com

\section{Research Article \\ Volume 2 Issue 1}

Received Date: December 06, 2017

Published Date: January 10, 2018

\section{Abstract}

Background: Silymarin, a widely used hepatoprotective drug, exhibits low oral bioavailability mainly because of poor water solubility, poor absorption, rapid metabolism and ultimately poor oral bioavailability of silymarin.

Objective: Therefore, the present study was aimed to investigate the effects of Piperine, Fulvic acid and Lysergol as bioenhancers on the intestinal absorption of silymarin.

Method: Non-everted sacs of rat small intestines were incubated in tyrode buffer solution which contained Silymarin in the absence or presence of various bioenhancers viz. Piperine, Fulvic acid and Lysergol alone and in combinations with each other in different concentrations.

Result: It was found that Silymarin alone had apparent permeability coefficient Papp x $10^{-6} \mathrm{~cm} / \mathrm{s}$ value of 1.63 and human fraction absorbed $F a(\%)$ value of $21 \%$ as compared to which the highest values Papp x 10-6 cm/s 7.01 and $F a(\%)$ $>100 \%$ were found to be for Silymarin- Fulvic acid (1:1) mixture + Piperine (10\%). The absorption transport of Silymarin was significantly enhanced in the presence of Piperine and Fulvic acid, suggesting that the Piperine acted as hepatic and intestinal glucoronidation inhibitor of Silymarin and Fulvic acid increased the water solubility of Silymarin and aided in the transport of Silymarin across the intestinal epithelial cells. However, the addition of Lysergol at various concentrations (1\%, 5\% and 10\%) had no significant effect on Silymarin transport.

Conclusion: The results of the study suggest that the use of such effective bioenhancers in Silymarin formulations may enhance the oral absorption and hence the oral bioavailability of Silymarin.

Keywords: Silymarin; Piperine; Fulvic acid; Lysergol; Permeability; Bioavailability; Solubility 


\section{Bioequivalence \& Bioavailability International Journal}

\section{Introduction}

Silymarin, a flavonolignan derived from Silybum marianum, Asteraceae, is a standardized extract consisting of approximately $70-80 \%$ Silymarin flavonolignans (Silybin A \& B, Isosilybin A \& B, Silydianin and Silychristin) and flavonoids (Taxifolin and Quercitin) and remaining $20-30 \%$ fraction of chemically undefined fractions [1]. It possesses diverse pharmacological activities, including hepatoprotective, antioxidant, antiinflammatory, anticancer, cardio protective etc [2]. The pharmacokinetic studies both in rats and humans have revealed poor absorption, rapid metabolism and ultimately poor oral bioavailability of Silymarin [3,4]. A lot of bioavailability enhancement approaches for Silymarin are available in literature like complexation with cyclodextrins, solid dispersion method, formation of micro and nanoparticles, liposomes, phytosomes and self emulsifying drug delivery systems are discussed extensively [1]. The most recent and newest approach is the use of bioavailability enhancer. The use of bioenhancer seems to be a fruitful approach for increasing the bioavailability of an orally administered drug and nutraceutical which leads to reduction in drug cost, toxicity and other adverse effects [5]. This research paper describes the role of various natural products along with their mechanism of action by virtue of which they might act as bioenhancers of Silymarin through permeability studies across non-everted gut sac of rat small intestine. Intestinal permeability is related to the ability of a compound to move across the epithelial barrier of the intestine and represents a direct measurement of the local absorption rate and reflects the transport velocity across the epithelial barrier, expressed as centimeter per second [6]. Several factors can affect the transfer process of drug compounds across the intestinal epithelial mucosa, such the physicochemical properties of the absorbed compound, physiological factors, formulation related factors etc [7]. The three groups of methods namely, in vivo, in situ and in vitro methods for investigating the principal mechanisms of absorption in animals are reported in literature. The choice of model depends totally on the questions to be answered with respect to the test compound being studied [8]. We opted out for the non-everted gut sac model as it is a simple and rapid technique for the study of intestinal drug transport for the evaluation of in vitro permeability in rats. It is capable of predicting both the extent and rate of drug absorption, providing information with regard to preabsorptive metabolism or degradation, thereby possibly predicting the human oral absorption of drugs from in vitro measured data. This model has advantages over everted gut sac model as preparation of sac was simple, the amounts of the drug required for the study are relatively small, samples can be collected successively and frequently, the collected samples are analytically clean which facilitates quantitative analysis, morphological damage caused by everting the intestinal tissue can be avoided and lastly, the time employed for the transport experiment may be longer than that of everted gut sac model [9]. We identified the primary reasons for poor oral bioavailability of Silymarin from the literature and targeted bioenhancers with suitable mechanism of actions. The bioenhancers selected were all of natural origin like Piperine, Fulvic acid and Lysergol (Figure 1). Ghosal S, have extensively described in the US Patent various examples where Fulvic acid is used as bioenhancers viz. purified Fulvic acid - glibenclamide drug delivery system, potentiation of anti-diabetic effect of insulin (p.o.) by purified Fulvic acid-insulin compositions and pentazocin (Ptz)-purified Fulvic acid carrier compositions etc [10-12]. Similarly, Piperine had been used along with a number of drugs because of its bioenhancing effects. Examples include Piperine and Aflatoxin B [13], Piperine and Phenytoin [14], Piperine and Pentobarbitone [15], Piperine and Curcumin [16], Piperine and $\beta$-Carotene [17], Piperine and Coenzyme $\mathrm{Q}_{10}$ [18], Piperine and Resveratrol [19] are evident from literature. Lysergol, a phytomolecule, is obtained from Morning Glory Plant (Ipomoea spp.) which enhances the killing activities of different antibiotics on bacteria and is a promising herbal bioenhancer. Lysergol enhanced the antimicrobial effect of the antibiotic compound in the range of 2-12-folds. Lysergol also improved the bioavailability of berberine after oral administration in Sprague-Dawley rats. The bioenhancement might be by inhibiting the metabolism of berberine or modifying its transport across cell membranes. More studies will have to be carried out to unearth the mechanism and establish the role of as a promising bioenhancer [20]. 


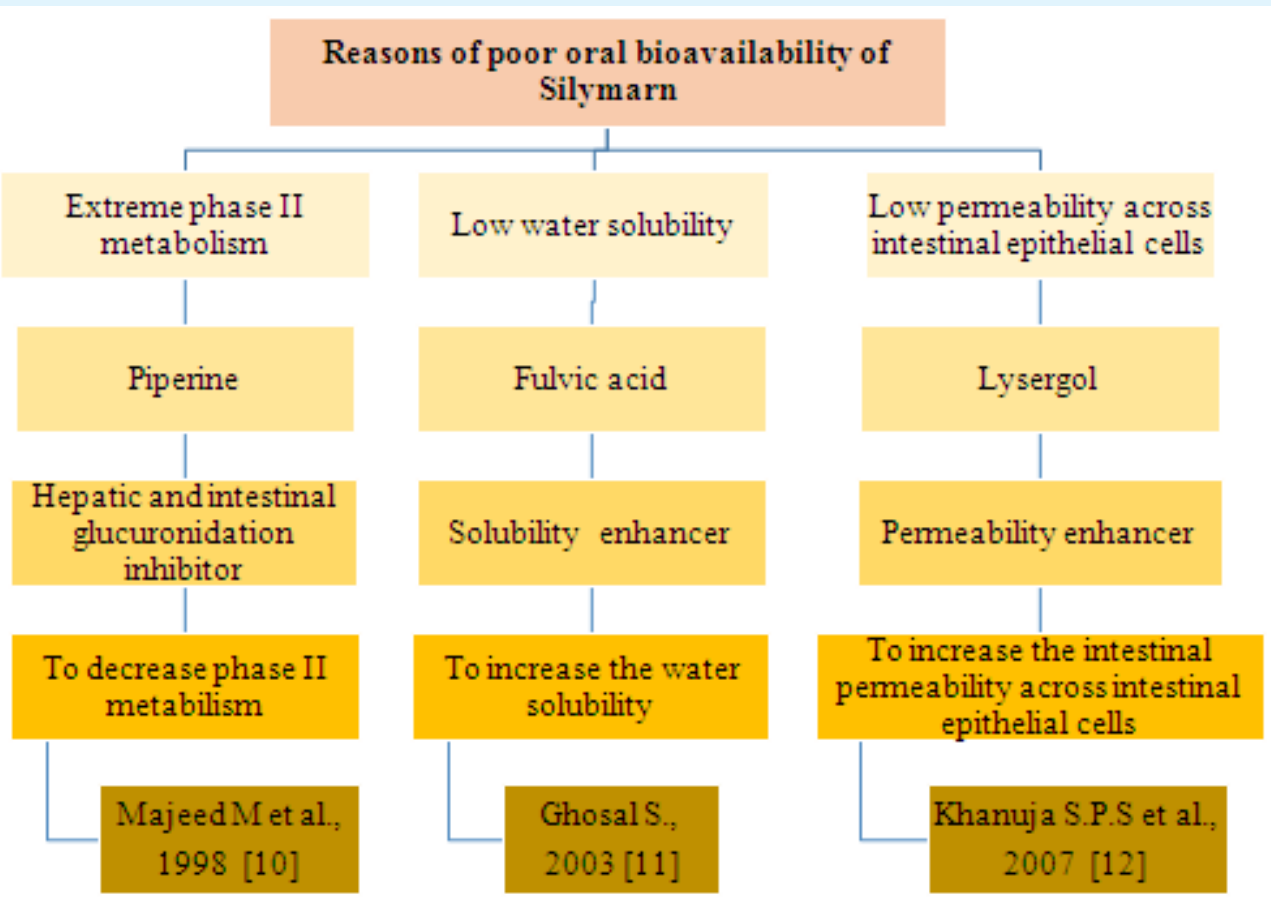

Figure 1: Mechanism of action of various bioenhancers .

\section{Methodology}

\section{Materials}

Silymarin $70 \%$ dry powder extract was obtained as a gift sample from Maneesh Pharmaceuticals Ltd., Mumbai, India, Piperine 98\% was purchased from Sigma Aldrich, New Delhi, Lysergol 97\% was obtained as a gift sample from Chemical Resources, Panchkula, Haryana, India and Fulvic acid was extracted from Shilajit capsules with the help of microwave assisted extraction technique Since standard Fulvic acid was not available to us, we characterized the extracted Fulvic acid by optical microscopy, UV-Vis spectroscopy, DSC, FT-IT, NMR, Elemental analysis by CHNS analyzer and X-ray diffraction techniques to confirm the chemical and structural integrity of Fulvic acid [21]. Double distilled water was used throughout the study where and when needed. Deionized water using Ionic interchanged columns MilliQ (Millipore) and Methanol HPLC grade (Fisher Scientific) were used as solvents for HPLC analysis of Silymarin samples. All reagents were of analytical grade unless indicated otherwise.

\section{Buffer solutions}

Tyrode solution: The $500 \mathrm{ml}$ of tyrode buffer contained: $\mathrm{NaCl}(4.0 \mathrm{~g}), \mathrm{KCl}(0.1 \mathrm{~g}), \mathrm{NaHCO}_{3}(0.5 \mathrm{~g}), \mathrm{CaCl}_{2}(0.1 \mathrm{~g})$,
$\left.\begin{array}{llllll}\mathrm{MgCl}_{2} & (0.09 & \mathrm{g}\end{array}\right), \quad \mathrm{NaH}_{2} \mathrm{PO}_{4} \quad(0.025 \mathrm{~g})$, glucose $\quad(0.5 \mathrm{~g})$ dissolved in $500 \mathrm{ml}$ of double distilled water.

0.9\% Normal saline: $0.9 \mathrm{~g}$ of $\mathrm{NaCl}$ was dissolved in 100 $\mathrm{ml}$ double distilled water.

\section{Methods}

\section{Analytical Method}

The amount of Silymarin (Silybin) outside the gut sac in the serosal medium was assayed using HPLC. An isocratic mobile phase was used for analysis which consisted of methanol and water $(1 \% \mathrm{v} / \mathrm{v}$ acetic acid) in the ratio 55:45, and a flow rate of $1 \mathrm{~mL} / \mathrm{min}$ and injection volume $20 \mu \mathrm{L}$. The detective wavelength was set at $288 \mathrm{~nm}$. The concentration of Silymarin (Silybin) from mucosal to serosal surfaces across the intestine was measured by collecting samples from the serosal medium periodically at different time intervals $0,15,30,45,60$ and 90 minutes and analyzed by HPLC-UV. Quantification of Silymarin (Silybin) was carried out by measuring the peak areas in relation to calibration plot chromatograms under the same conditions [22].

\section{Rat Gut-Sac Apparatus}

The complete set-up, glass assembly and other parts used in the present rat gut sac in-vitro permeability study are shown in Figure 2. The specially designed and 
fabricated glass assembly consisted of a cylindrical shape vessel (length $20 \mathrm{~cm}$ and internal diameter $3.5 \mathrm{~cm}$ ) with jacketed walls for the maintenance of the temperature at $37^{\circ} \mathrm{C} \pm 0.5$. In the glass vessel tapering at each end were available for inlet and outlet pipes which were connected to the water bath. Thus, by circulating water at controlled temperature through the jacket, the temperature of the gut sac and the tyrode medium could be controlled. On the top of glass vessel a rubber cork was placed which provides access for three different things viz. tube 1 for injecting the various test samples inside the intestinal sac (i.e. the injecting syringe and needle), tube 2 for sampling from outside the sac (i.e. sampling tube) and tube 3 a pipe known as aerator pipe which is also dropped inside the glass vessel for proper aeration of the tissue. For mounting the rat intestinal tissue in order to form a sac, the tissue was ligated from the proximal end with the tube 1 and suspended and the distal end of the tissue was ligated with a 1 gm glass weight to make an empty gut sac, to prevent peristaltic muscular contractions, which may otherwise alter the shape and internal volume of the sac and to keep the sac in vertical position. The $1 \mathrm{~g}$ glass weight was the minimum weight to secure the abovementioned conditions and to prevent the sac septum to become thin. A magnetic stirrer was placed inside the glass apparatus for the proper stirring and maintenance of sink conditions. This glass apparatus was clamped on the laboratory stand beneath which the magnetic stirrer was placed, the aerator pipe was adjusted properly, the inlet and outlet pipes of the water jacket were connected to the water bath to maintain the temperature up to $37^{\circ} \mathrm{C}$ and temperature was monitored with the help of a thermometer and all the electrical appliances were connected to the power.

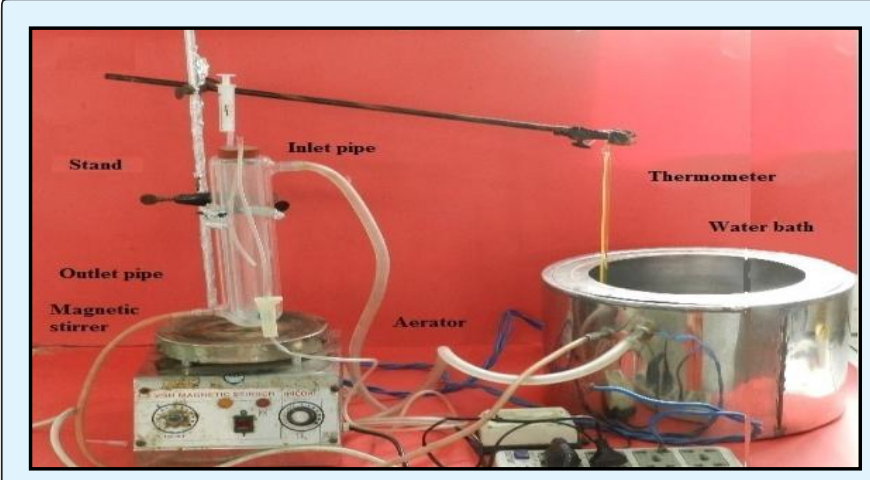

Figure 2: Complete assembly of the rat gut sac apparatus.

\section{Preparation of Non-Everted Intestinal Sacs}

The animals were hygienically kept in a controlled environment in large polypropylene cages at air conditioned temperature $\left(20-25^{\circ} \mathrm{C}\right)$ with a $12 \mathrm{~h}$ light/dark cycle in Central Animal House, Jamia Hamdard, New Delhi (India). For the experimental purpose, male wistar rats, weighing approximately 250-300 g, fasted over night with free access to water were used in this study. Upon anesthesia, a midline longitudinal incision of 3-4 cm was made in the abdomen and the small intestine was located. The intestine was cut and clean ileum of approximately $5 \mathrm{~cm}$ after removing underlying mesenterium was contained in fresh tyrode buffer $(\mathrm{pH}$ 7.4) at $37^{\circ} \mathrm{C}$. Sacs were then washed with $0.9 \% \mathrm{NaCl}$ with the help of $5 \mathrm{~mL}$ syringe to remove animal waste if present and oxygenated with the help of aerator. Next the non-everted gut sac $(5 \mathrm{~cm})$ prepared from rat ileum which can accommodate $1 \mathrm{~mL}$ of test solution was immediately mounted on the specially designed jacketed glass assembly containing $50 \mathrm{~mL}$ of tyrode buffer maintained at $37{ }^{\circ} \mathrm{C}$. The aeration was provided to the tissue with the help of aerator and the tyrode buffer was stirred with the help of magnetic bead at $50 \mathrm{rpm}$. The sac was mounted/ hung vertically in the glass assembly and the lower end was tied with a small weight attached to it. After mounting the sac in the assembly and its stabilization in the experimental conditions for 5-10 minutes, the sac was then filled with $1 \mathrm{~mL}$ of drug solution with the help of a long blunt needle. The solutions $(2 \mathrm{~mL})$ from outside the sac i.e. from serosal side were taken at intervals $0,15,30,45,60$ and 90 minutes and replaced with fresh tyrode buffer $(2 \mathrm{~mL})$. The obtained samples were analyzed by HPLC-UV for the transport of drug from mucosal side to serosal side in 90 minutes. This method was the modification of non-everted rat intestinal sac model provided in the literature [9].

\section{Preparation of Various Drug-Bioenhancer Compositions}

To examine the effect of Fulvic acid, Piperine and Lysergol on the intestinal permeability of Silymarin across rat ileum, the mucosal medium was filled with tyrode solution containing various drug-bioenhancer concentrations as shown in Table 1. At appropriate time points, samples were withdrawn from the serosal medium and replaced with fresh tyrode solution. The contents of the samples were subjected to HPLC analysis. The permeability values (Papp) so obtained when Silymarin was used in combination with various bioenhancers were compared to the permeability value (Papp) of Silymarin alone in order to establish the roles of Fulvic acid, Piperine and Lysergol as bioenhancers of Silymarin in terms of the folds increase in absorption, Papp $\left(\mathrm{x} 10^{-6} \mathrm{~cm} / \mathrm{s}\right)$ and human fraction $F a(\%)$. 


\begin{tabular}{|c|c|}
\hline S. No & Test solution \\
\hline & Silymarin+ Lysergol $(1,5 \& 10 \% \mathrm{w} / \mathrm{w})$ \\
& Silymarin+ Piperine $(1,5 \& 10 \% \mathrm{w} / \mathrm{w})$ \\
1 & Silymarin+ Piperine $(10 \%)+$ Lysergol $(10 \%)$ \\
2 & Silymarin+ Fulvic acid $(1: 1 \mathrm{w} / \mathrm{w})$ mixture \\
3 & Silymarin+ Fulvic acid $(1: 1 \mathrm{w} / \mathrm{w})$ mixture + \\
4 & Piperine $(10 \%)$ \\
5 & Silymarin+ Fulvic acid $(1: 1 \mathrm{w} / \mathrm{w})$ mixture + \\
6 & Lysergol $(10 \%)$ \\
7 & Silymarin+ Fulvic acid $(1: 1 \mathrm{w} / \mathrm{w})$ mixture + \\
& Piperine (10\%) + Lysergol (10\%) \\
\hline
\end{tabular}

Table 1: Various drug-bioenhancer combinations tested to study the effect of bioenhancers on the absorption of Silymarin.

\section{Histological studies}

Histological studies were performed to evaluate the effect of drug and bioenhancers on the intestinal sac models during the transport. Before and after the experiment, some intestinal sacs were removed. The specimens were immediately fixed in $10 \%$ formaldehydesaline solution and embedded in paraffin. Then 2-4 micrometer sections were stained with hematoxylin and eosin ( $\mathrm{H}$ and $\mathrm{E}$ ) for histological evaluation [9].

\section{Results}

\section{Calculation of the apparent permeability coefficient and interpretation of results}

The apparent permeability coefficient (Papp) of Silymarin under various conditions was calculated from the following equation:

$$
P_{\text {app }}=\frac{\mathrm{dQ}}{\mathrm{dt}} \cdot \underline{\underline{1}}
$$

Where; Papp (cm/s) is the apparent permeability coefficient, $d Q / d t(\mu \mathrm{g} / \mathrm{s})$ is the steady state appearance rate on the acceptor solution or the amount of drug transported across the membrane per unit of time, $A$ $\left(\mathrm{cm}^{2}\right)$ is the surface area of the intestinal sacs available for permeation and $C o(\mu \mathrm{g} / \mathrm{mL})$ is the initial concentration of drug inside the sacs. The surface area $\mathrm{A}\left(\mathrm{cm}^{2}\right)$ of intestinal sac assuming to have a cylindrical shape with length $5 \mathrm{~cm}$ and diameter $0.5 \mathrm{~cm}$ was calculated as $8.2425 \mathrm{~cm}^{2}$ per sac [9].

\section{Calibration Plot of Silymarin in Tyrode Buffer Containing $0.5 \% \mathrm{w} / \mathrm{v}$ of Sodium Lauryl Sulphate}

Silymarin suffer from poor aqueous solubility and its inability to directly go into the tyrode solution for making the standard plot in HPLC lead us to use of sodium lauryl sulphate as a solubilizer [21]. Different concentrations in the range $(0-40 \mu \mathrm{g} / \mathrm{ml})$ of Silymarin in the tyrode buffer solution containing $0.5 \% \mathrm{w} / \mathrm{v}$ SLS were prepared for the calibration plot and the peak areas were obtained at different concentrations. The areas of the peak were plotted against the concentration of drug and a linear calibration plot in the range with $r^{2}=0.9947$ was obtained with HPLC method (Figure 3). The slope of the plot was used to calculate the Silymarin (Silybin) concentration in the unknown samples. The retention time of Silymarin (Silybin A \& B) were 11.667 and 13.163 minutes respectively (Figure 4). The quantification of Silymarin was carried out by measuring the peak areas of Silybin A + Silybin B in relation to calibration plot chromatograms. It was found that under the set chromatographic conditions, Piperine, Lysergol, Fulvic acid did not interfere with the assay of Silymarin (Silybin).

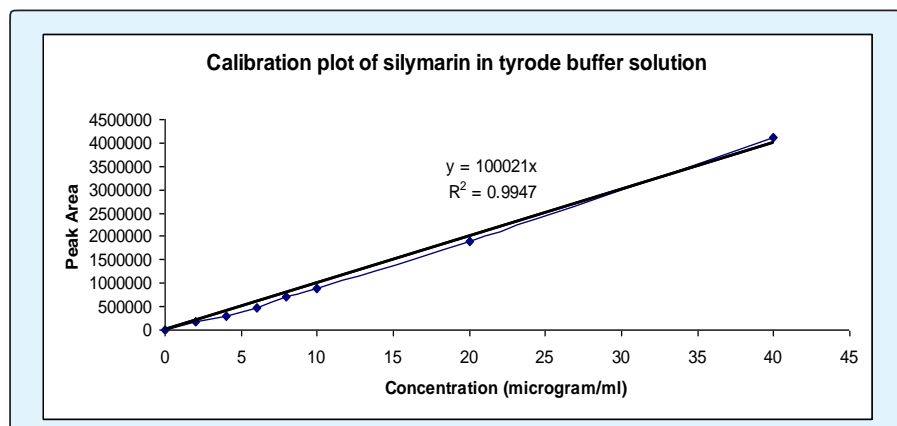

Figure 3: Area versus concentration graph of Silymarin in tyrode buffer solution.

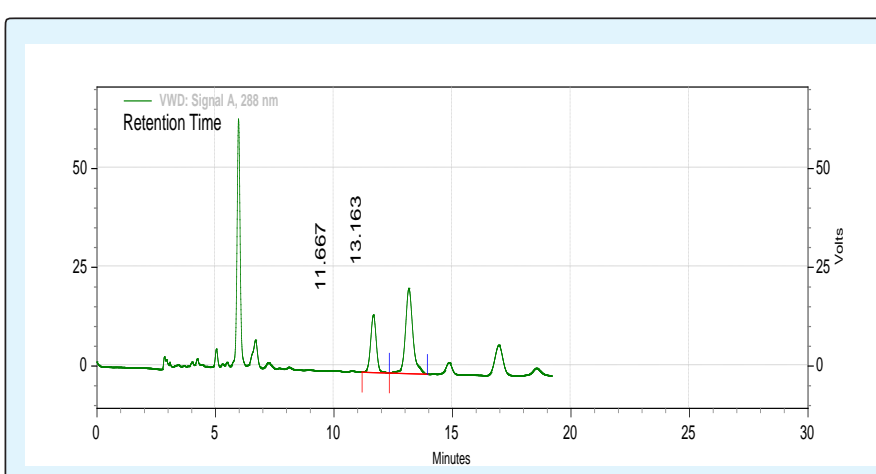

Figure 4: Chromatogram for the separation of drug peak. 


\section{Bioequivalence \& Bioavailability International Journal}

\section{Concentration Dependent Absorption Profile of Silymarin}

First, intestinal permeability of Silymarin alone was tested in tyrode solution across the rat ileum at various concentrations $(10,25,50,100,200 \mu \mathrm{g} / \mathrm{mL})$ to observe the concentration dependent absorption profile of silymarin. At the $10 \mu \mathrm{g} / \mathrm{ml}$, the Silymarin (Silybin) was below the detection limit in the serosal compartment throughout the time period. This could be attributed to very low concentration inside the gut sac. Concentration dependent absorption changes were seen at 25- 200 $\mu \mathrm{g} / \mathrm{ml}$ as shown in Table 2 . The increase in absorption was seen in the order $25<50<100<200 \mu \mathrm{g} / \mathrm{ml}$. But no major absorption difference was observed at higher concentrations i.e. 100 and $200 \mu \mathrm{g} / \mathrm{ml}$ or that simply uptake did not increase with increase in concentration after a certain level. This can be due to the saturation of absorption and transport at these concentrations (Figure 5). Therefore, Silymarin concentration $(100 \mu \mathrm{g} / \mathrm{ml})$ was selected for the further comparative studies with bioenhancers in order to understand the effect of bioenhancer on the absorption profile of Silymarin.

\begin{tabular}{|c|c|c|c|}
\hline S. No & Initial Concentration $(\boldsymbol{\mu g} / \mathbf{m l})$ & Concentration at $\mathbf{6 0}$ min incubation $(\boldsymbol{\mu g} / \mathbf{m l})$ & Absorption $(\boldsymbol{\mu g} / \mathbf{c m})$ \\
\hline 1 & 10 & Below the detection limit & -- \\
\hline 2 & 25 & 1.76 & 0.352 \\
\hline 3 & 50 & 3.37 & 0.674 \\
\hline 4 & 100 & 7.29 & 1.458 \\
\hline 5 & 200 & 7.85 & 1.570 \\
\hline
\end{tabular}

Table 2: Concentration dependent absorption of the Silymarin in tyrode solution

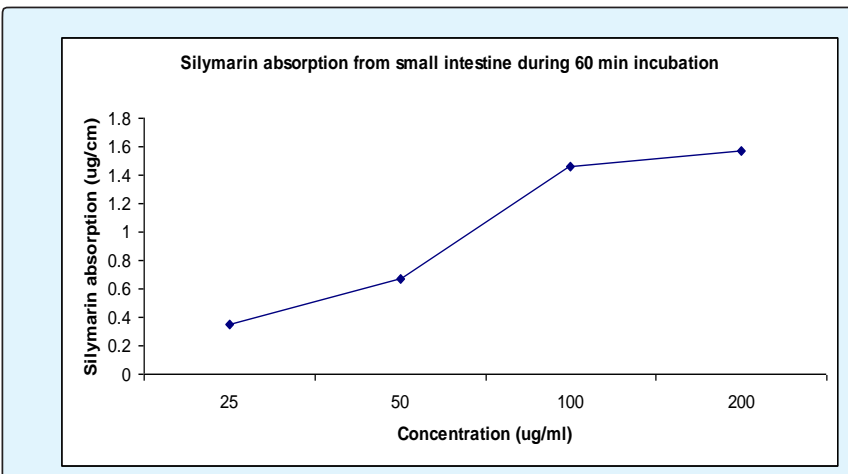

Figure 5: Concentration dependant absorption profile of Silymarin in non-everted rat gut sac tissue.

\section{Intestinal Permeability Studies}

The results of the study are summarized in Table 3 and Figure 6 in terms of folds increase in Permeability, Apparent Permeability values and Human Fraction Absorbed.

\begin{tabular}{|c|c|c|c|c|c|c|c|c|c|c|}
\hline Time (min) & \multicolumn{10}{|c|}{ Concentration of drug- bioenhancer combinations $(\mu \mathrm{g} / \mathrm{ml})$} \\
\hline & \begin{tabular}{|c|} 
Sily \\
alone
\end{tabular} & \begin{tabular}{|c} 
Sily-FA \\
$1: 1$ \\
mixture
\end{tabular} & $\begin{array}{c}\text { Sily-Pip } \\
(5 \%)\end{array}$ & $\left|\begin{array}{c}\text { Sily-Pip } \\
(10 \%)\end{array}\right|$ & $\begin{array}{c}\text { Sily-Lys } \\
(5 \%)\end{array}$ & $\begin{array}{l}\text { Sily-Lys } \\
(10 \%)\end{array}$ & $\mid \begin{array}{c}\text { Sily-FA 1:1 } \\
\text { mixture } \\
+ \text { Pip (10\%) }\end{array}$ & $\begin{array}{c}\text { Sily-FA 1:1 } \\
\text { mixture + Lys } \\
(10 \%)\end{array}$ & $\begin{array}{c}\text { Sily + Pip (10\%) + } \\
\text { Lys }(10 \%)\end{array}$ & $\begin{array}{c}\text { Sily-FA 1:1 } \\
\text { mixture + Pip } \\
(10 \%)+\text { Lys } \\
(10 \%)\end{array}$ \\
\hline 0 & 0 & 0 & 0 & 0 & 0 & 0 & 0 & 0 & 0 & 0 \\
\hline 15 & 1.44 & 4.43 & 2.12 & 3.06 & 1.51 & 1.79 & 6.13 & 3.98 & 2.85 & 5.68 \\
\hline 30 & 2.80 & 6.65 & 4.95 & 6.16 & 3.01 & 3.65 & 11.2 & 5.97 & 5.65 & 12.12 \\
\hline 45 & 4.11 & 13.4 & 7.73 & 9.88 & 5.50 & 6.12 & 17.85 & 12.56 & 8.02 & 18.65 \\
\hline 60 & 6.80 & 19.9 & 10.11 & 14.21 & 7.23 & 7.89 & 26.6 & 18.67 & 13.21 & 23.45 \\
\hline 90 & 7.29 & 23.97 & 13.85 & 18.88 & 8.25 & 9.80 & 31.18 & 23.35 & 16.11 & 29.12 \\
\hline Folds & -- & 3.28 & 1.90 & 2.58 & 1.13 & 1.35 & 4.20 & 3.20 & 2.21 & 3.99 \\
\hline $\mathrm{Papp}\left(\mathrm{x} 10^{-6} \mathrm{~cm} / \mathrm{s}\right)$ & 1.63 & 5.38 & 3.11 & 4.24 & 1.85 & 2.20 & 7.01 & 5.24 & 3.62 & 6.54 \\
\hline$F a(\%)^{*}$ & $21 \%$ & $60-80 \%$ & $55 \%$ & $60 \%$ & $21-35 \%$ & $35 \%$ & $>100$ & $60-80 \%$ & $55 \%$ & $90 \%$ \\
\hline
\end{tabular}

* Values as reported in the literature [9]

Table 3: Apparent permeability values of drug-bioenhancer combinations. 


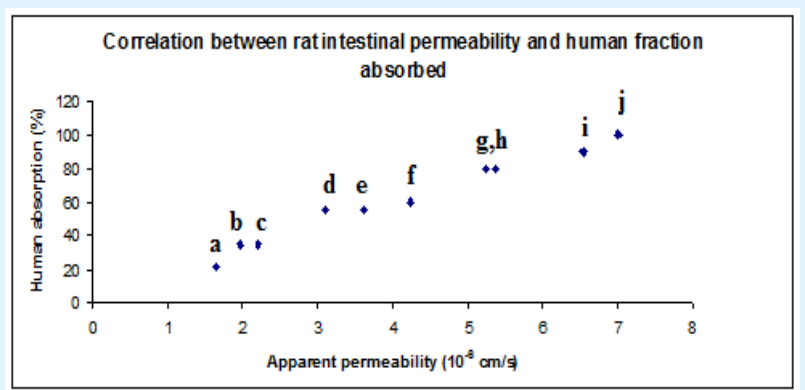

Figure 6: Correlation between rat intestinal permeability and human fraction absorbed. a- Sily alone, b- Sily-Lys (5\%), c- Sily-Lys (10\%), d- Sily-Pip (5\%), e- Sily- Pip $(10 \%)$ - Lys (10\%), f- Sily-Pip (10\%), g- Sily-FA 1:1 mixture + Lys (10\%), h- Sily-FA (1:1) mixture, i- Sily-FA 1:1 mixture + Pip (10\%) + Lys (10\%), j- Sily-FA (1:1) mixture + Pip (10\%)

Silymarin alone: In the rat intestinal study conducted on Silymarin $(100 \mu \mathrm{g} / \mathrm{ml})$ alone for a period of 90 minutes, the apparent permeability coefficients Papp $(\mathrm{cm} / \mathrm{s})$ was found to be $1.63 \times 10^{-6} \mathrm{~cm} / \mathrm{s}$ and the estimated human fraction absorbed $\mathrm{Fa}(\%)$ was $21 \%$. Hence it was concluded that Silymarin alone exhibited low permeability across the rat gut sac tissue and the reasons were attributed to the poor water solubility of silymarin.

Silymarin- Fulvic acid (1:1) mixture: The Papp (cm/s) value for binary mixture of silymarin-Fulvic acid (1:1) mixture was found to be $5.38 \times 10^{-6} \mathrm{~cm} / \mathrm{s}$ which was much higher than Silymarin alone and 3.28 folds increase in permeability of Silymarin was seen with Fulvic acid. This increase was attributed to the water solubility enhancement of Silymarin by Fulvic acid and thus confirmed the importance of Fulvic acid.

Silymarin- Piperine: The rat intestinal permeability of Silymarin in the presence of Piperine as a bioavailability enhancer was conducted at various concentrations of Piperine $(1 \%, 5 \%$ and $10 \% \mathrm{w} / \mathrm{w}$ of active drug). No noticeable change in the Papp $(\mathrm{cm} / \mathrm{s})$ was seen when Piperine $(1 \% \mathrm{w} / \mathrm{w})$ was used as a bioenhancer of Silymarin as compared to Silymarin alone. Whereas, in case of Piperine $(5 \%)$ and $(10 \%)$ the Papp values of Silymarin were found to be $3.11 \times 10^{-6}$ and $4.24 \times 10^{-6} \mathrm{~cm} / \mathrm{s}$ with permeability enhancement of 1.90 and 2.58 folds respectively. This can be attributed to the strong P-gp inhibition and intestinal glucoronidation inhibition by Piperine at these concentrations which might have stopped the efflux mechanism or metabolic conversion of Silymarin during its transport across the rat intestinal cells. Out of the three concentrations tested, only Piperine $10 \%$ was carry forward for further studies.
Silymarin- Lysergol: Experiment was conducted in the presence of Lysergol at various concentrations $(1 \%, 5 \%$, $10 \% \mathrm{w} / \mathrm{w}$ of active drug). No noticeable change in the Papp (cm/s) was seen when Lysergol $(1 \% \mathrm{w} / \mathrm{w})$ was used as a bioenhancer of Silymarin as compared to Silymarin alone. Whereas, in case of Lysergol (5\%) and (10\%) the Papp values of Silymarin were found to be $1.85 \times 10^{-6}$ and $2.20 \times 10^{-6} \mathrm{~cm} / \mathrm{s}$ with permeability enhancement of 1.13 and 1.35 folds respectively. No major difference was observed between the two concentrations of Lysergol. From this data, the estimated human fraction absorbed was found to be $21-35 \%$ and $35 \%$ respectively. And based on the results Lysergol (10\%) was carry forward for further studies.

Silymarin- Piperine- Lysergol: After individually testing the effect of Piperine and Lysergol on the rat intestinal permeability of silymarin, the two bioenhancers were tried in combination with each other (in the concentration of $10 \% \mathrm{w} / \mathrm{w}$ of active drug) for any synergistic effects. Thus, for Silymarin $(100 \mu \mathrm{g} / \mathrm{ml})$ Piperine $(10 \%)$ - Lysergol $(10 \%)$ combination the Papp $\left(\times 10^{-6} \mathrm{~cm} / \mathrm{s}\right)$ of Silymarin was found to be $3.62 \times 10^{-6} \mathrm{~cm} / \mathrm{s}$ and a 2.21 folds increase in permeability was observed in this case in comparison to Silymarin alone. Here, no synergistic effects were seen between Piperine (10\%) and Lysergol (10\%) in combination.

Silymarin- Fulvic acid (1:1) mixture + Piperine (10\%): Further a combination of silymarin- Fulvic acid (1:1) mixture with Piperine (10\%) was tested and the Papp $(\mathrm{cm} / \mathrm{s})$ was found to be $7.01 \times 10^{-6} \mathrm{~cm} / \mathrm{s}$ and a 4.20 folds increase in permeability of Silymarin at a period of 90 minutes was observed as compared to Silymarin alone. This was highest amongst all groups.

Silymarin- Fulvic acid (1:1) mixture + Lysergol (10\%): In this case the Silymarin- Fulvic acid (1:1) mixture was tested with Lysergol (10\%), and the Papp $\left(\mathrm{cm} / \mathrm{s}\right.$ ) of Silymarin was found to be $5.24 \times 10^{-6} \mathrm{~cm} / \mathrm{s}$ and a 3.20 folds increase in permeability was seen as compared to Silymarin alone. The estimated human fraction absorbed was $60-80 \%$ and these results were more or less similar to Silymarin- Fulvic acid (1:1) mixture and no synergistic effect between Fulvic acid and Lysergol was observed here.

Silymarin- Fulvic acid (1:1) mixture + Piperine (10\%) + Lysergol (10\%): Lastly, after optimizing the individual bioenhancer concentrations, all the three bioenhancers were used in combination in the concentrations in which they have previously shown their best permeability results for silymarin. Thus, silymarin- Fulvic acid (1:1) mixture with Piperine (10\%) and Lysergol (10\%) was tested for its effect on the rat intestinal permeability of Silymarin and compared the results with Silymarin alone. The Papp $(\mathrm{cm} / \mathrm{s})$ in combination was found to be $6.54 \mathrm{x}$ 
$10^{-6} \mathrm{~cm} / \mathrm{s}$ as compared to $1.63 \times 10^{-6} \mathrm{~cm} / \mathrm{s}$ of Silymarin alone. A 3.99 folds increase in permeability of Silymarin was achieved which was higher than Silymarin alone but was found to be less than Silymarin- Fulvic acid (1:1) mixture + Piperine (10\%) combination.

\section{Histological Studies}

Histological studies were performed to evaluate the effect of drug and bioenhancers on the non-everted intestinal sac models during the transport. After the experiment, some intestinal sacs were removed. The specimens were immediately fixed in $10 \%$ formaldehydesaline solution and embedded in paraffin. 2-4 micrometer sections were stained with hematoxylin and eosin ( $\mathrm{H}$ and E) for histological evaluation. The light microscopic pictures of the normal intestinal sacs were obtained and compared with intestinal tissue treated with bioenhancers. It was apparent that no significant presence of inflammatory cells was observed in bioenhancer treated intestinal sacs. No disruption of cellular integrity and changes in the structure of the intestines was observed as shown in Figure 7.

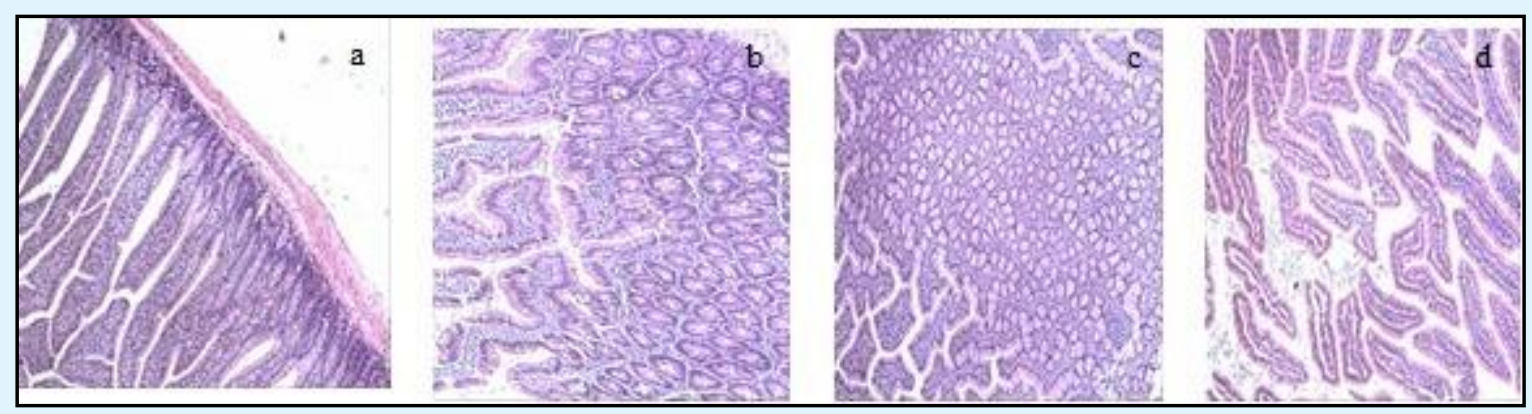

Figure 7: Histological section of rat intestine: Control (a) Piperine (b) Lysergol (c) Fulvic acid (d) (H and E stain, 100 -fold magnification).

\section{Discussion}

In this study, the effect of bioenhancers on the permeability of Silymarin was studied extensively through non-everted rat gut sac model. We attempted to improve the intestinal absorption of Silymarin by enhancing its solubility, permeability and by modulating the intestinal metabolizing enzymes. Non-everted rat gut sac model was chosen because it is simple, rapid and economic method. Histological studies were conducted to confirm the validity of this in vitro model and intestinal tissue was observed by light microscopy after 90 minutes of experiment which showed that the intestine remained intact morphologically throughout the experiment. Ileum excision was made in anesthetized rats before they were killed by cervical dislocation to avoid the deterioration of tissues and active transport. First, permeability studies were conducted for various concentrations of Silymarin $(10,25,50,100$ and $200 \mu \mathrm{g} / \mathrm{ml})$ to study the concentration dependant absorption of Silymarin. It was seen that increase in concentration at a certain level i.e. from 100 to $200 \mu \mathrm{g} / \mathrm{ml}$ did not increase the absorption of Silymarin in $5 \mathrm{~cm}$ long gut sac tissue and thus the Silymarin $(100 \mu \mathrm{g} / \mathrm{ml})$ was selected for further studies. Permeability studies were than conducted for drug - bioenhancers combinations at various concentrations and (Figure 8) shows the increase in permeability in the range 1.13 -
4.20 folds in presence of various bioenhancers as compared to Silymarin alone. Hence it can be concluded that: Enhancement in Silymarin absorption was obtained by using all the three bioenhancers as compared to the plain Silymarin dispersion at $100 \mu \mathrm{g} / \mathrm{ml}$ concentration in the order Fulvic acid >Piperine>Lysergol. Fulvic acid increased the solubility of Silymarin significantly that aided in the absorption. In a separate published study Javed et al. 2016 concluded that the solubility and dissolution were found to increase when Silymarin was complexed with Fulvic acid by physical mixture and kneading methods, establishing the role of Fulvic acid as a solubilizer of Silymarin [23]. Piperine concentrations were tested in the range $1 \%, 5 \%$ and $10 \%$ and it was observed that the absorption increased with the increase in concentration of the bioenhancers. Piperine being a known hepatic and intestinal glucoronidation inhibitor might have inhibited the phase II metabolism of Silymarin thus, inhibiting or reducing the rate of biotransformation of drug in the intestine. Bioenhancers were also tried in combination and the best results were shown by Silymarin: Fulvic acid 1:1 complex along with Piperine (10\%). And finally 1.35, 2.58, 3.28 and 4.20 folds increase in absorption of Silymarin was achieved with Lysergol (10\%), Piperine (10\%), Fulvic acid (1:1) and combination of Fulvic acid and Piperine (10\%) respectively. 


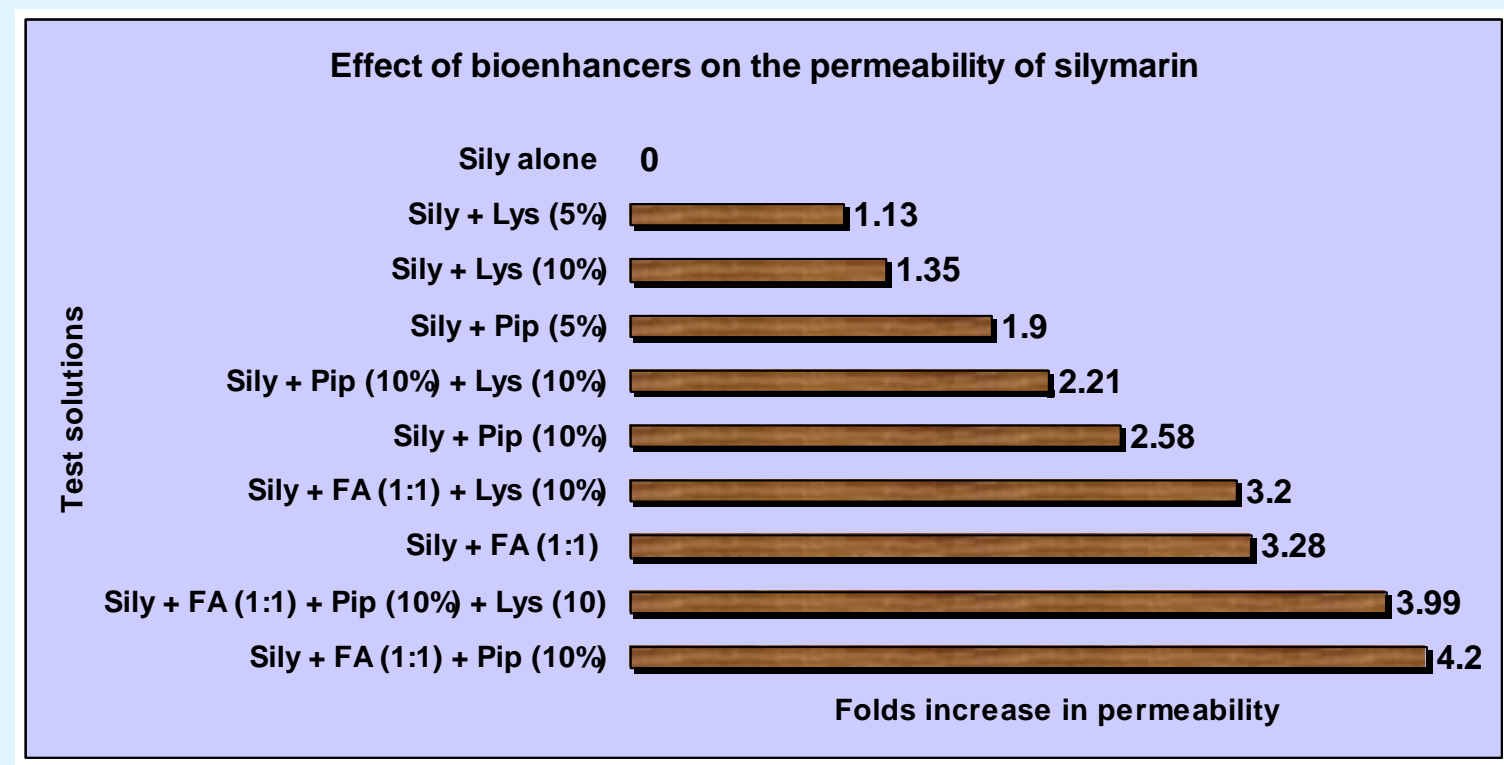

Figure 8: Effect of bioenhancer concentrations versus folds increase permeability of Silymarin.

\section{Conclusions}

Silymarin, a widely used hepatoprotective drug, exhibits low oral bioavailability mainly because of poor water solubility, poor absorption and rapid metabolism. Therefore, the present study was aimed to investigate the effects of bioenhancers like Piperine, Fulvic acid and Lysergol on the intestinal absorption of Silymarin. The results of the study suggested that the appropriate use of such effective bioenhancers in Silymarin formulations may enhance the oral absorption and hence the oral bioavailability of Silymarin.

\section{Acknowledgements}

S. Javed wishes to thank the Indian Council of Medical Research, New Delhi, India for providing financial assistance in the form of Senior Research Fellowship. Maneesh Pharmaceuticals Pvt. Ltd. India for providing the Silymarin $70 \%$ extract and Jamia Hamdard for the scientific ambience and environment for carrying out the studies.

\section{References}

1. Javed S, Kohli K, Ali M (2011) Reassessing bioavailability of Silymarin. Alt Med Rev 16(3): 239249.

2. Pradhan SC, Girish C (2006) Hepatoprotective herbal drug, Silymarin from experimental pharmacology to clinical medicine. Indian J Med Res 124(5): 491-504.
3. Wu JW, Lin LC, Hung SC, Chi CW, Tsai TH, et al. (2007) Analysis of silibinin in rat plasma and bile for hepatobiliary excretion and oral bioavailability application. J Pharm Biomed Anal 45(4): 635-641.

4. Wen Z, Dumas TE, Schrieber SJ, Hawke RL, Fried MW, et al. (2008) Pharmacokinetics and metabolic profiles of free, conjugated and total Silymarin flavonolignans in human plasma after oral administration of milk thistle extract. Drug Metab Dispos 36(1): 65-72.

5. Javed S, Ahsan W, Kohli K (2016) The concept of bioenhancers in bioavailability enhancement of drugs - a patent review. J Scient Lett 1(3): 143-165.

6. Nagare N, Damre A, Singh KS, Mallurwar SR, Iyer S, et al. (2010) Determination of Site of Absorption of Propranolol in Rat Gut Using In Situ Single-Pass Intestinal Perfusion. Indian J Pharm Sci 72(5): 625629.

7. Ferrec EL, Chesne C, Artusson P, Brayden D, Fabre G, et al. (2001) In Vitro Models of the Intestinal Barrier. The report and recommendations of ECVAM Workshop 46. European Centre for the Validation of Alternative methods. Altern Lab Anim 29(6): 649668.

8. Buckley ST, Fischer SM, Fricker G, Brandl M (2012) In vitro models to evaluate the permeability of poorly soluble drug entities: Challenges and perspectives. Eur J Pharm Sci 45(3): 235-250. 
9. Ruan LP, Chen S, Yu BY, Zhu DN, Cordell GA, et al. (2006) Prediction of human absorption of natural compounds by the non-everted rat intestinal sac model. Eur J Med Chem 41(5): 605-610.

10. Majeed M, Badmaev V, Rajendran R (1998) Use of Piperine as a bioavailability enhancer. United States Patent Number, 5744161.

11. Ghosal S (2003) Delivery system for pharmaceutical, nutritional and cosmetic ingredients, United States Patent 6558712 B1.

12. Khanuja SPS, Arya JS, Srivastava SK, Ajit KS, Tiruppadiripuliyur RK et al. (2007) Antibiotic pharmaceutical composition with Lysergol as bioenhancer and method of treatment. United States Patent Number: 20070060604A1.

13. Allameh A, Saxena M, Biswas G, Raj HG, Singh J, et al. (1992) Piperine, a plant alkaloid of the piper species, enhances the bioavailability of aflatoxin $B_{1}$ in rat tissues. Cancer Lett 61(3): 195-199.

14. Bano G, Amla V, Raina RK, Zutshi U, Chopra CL (1987) The effect of Piperine on pharmacokinetics of phenytoin in healthy volunteers. Planta Medica 53(6): 568-569.

15. Mujumdar AM, Dhuley JN, Deshmukh VK, Raman PH, Thorat SL, et al. (1990) Effect of Piperine on pentobarbitone induced hypnosis in rats. Indian J Exp Biol 28(5): 486-487.

16. Shoba G, Joy D, Joseph T, Majeed M, Rajendran R, et al. (1998) Influence of Piperine on the pharmacokinetics of curcumin in animals and human volunteers. Planta Medica 64(4): 353-356.
17. Badmaev V, Majeed $M$ and Norkus EP (1999) Piperine, an alkaloid derived from black pepper increases serum response of beta-carotene during 14days of oral beta-carotene supplementation. Nutr Res $19(3): 381-388$.

18. Badmaev V, Majeed M and Prakash L (2000) Piperine derived from black pepper increases the plasma levels of coenzyme $\mathrm{Q}_{10}$ following oral supplementation. J Nutr Biochem 11(2): 109-113.

19. Johnson JJ, Nihal M, Siddiqui IA, Scarlett CO, Bailey $\mathrm{HH}$, et al. (2011) Enhancing the bioavailability of resveratrol by combining it with Piperine. Mol Nutr Food Res 55(8): 1169-1176.

20. Patil S, Dash RP, Anandjiwala S, Nivsarkar M (2012) Simultaneous quantification of berberine and Lysergol by HPLC-UV: evidence that Lysergol enhances the oral bioavailability of berberine in rats. Biomed Chromatography 26(10): 1170-1175.

21. Javed S, Kohli K, Ali M (2013) Microwave-Assisted Extraction of Fulvic Acid from a Solid Dosage Form: A Statistical Approach. Journal of Pharmaceutical Innovation 8 (3): 175-186.

22. Javed S, Kohli K, Ali M (2012) Solid state compatibility between Silymarin and tablet excipients by thermal and non-thermal methods, its $\mathrm{pH}$ and solubility analysis. J Pharm Res 5(3): 1300-1305.

23. Javed S, Kohli K, Ahsan W (2016) Solubility and dissolution enhancement of Silymarin with Fulvic acid carrier. Int J Drug Dev \& Res 8: 9-14. 